\title{
Piano bass strings with reduced inharmonicity: theory and experiments
}

\author{
Jean-Pierre Dalmont* \\ Laboratoire d'acoustique de l'Université du Mans (LAUM, UMR CNRS6613), Avenue Olivier Messiaen, 72085 Le Mans, France
}

Received 22 July 2020, Accepted 11 January 2021

\begin{abstract}
In modern straight pianos and half-grand pianos the inharmonicity due to the bending stiffness of the strings makes the sound of lower notes of poor quality. The question of whether this can be corrected has been addressed in a previous paper and it was shown on the basis of numerical simulations that this might be possible. In the present paper we show using analytical models that fixing a single mass near the end of the string is a simple and efficient solution. Indeed, the experiments show that theoretical predictions are relevant. So, in practice, on the basis of a simple formula involving the inharmonicity coefficient, it is easy to deduce the position of a given mass in order to reduce significantly the inharmonicity of the bass strings.
\end{abstract}

Keywords: Inharmonicity, Piano, String, Tuning

\section{Introduction}

The use of harmonic resonators with harmonic eigenfrequencies - here called harmonic resonators - is the basis of most musical instruments because the harmonicity is needed so that an unambiguous pitch is assigned to a periodic or quasi-periodic signal (i.e. with decreasing amplitude). Instrument makers therefore use naturally harmonic resonators such as homogeneous strings or cylindrical pipes. In other cases, they optimize a geometry that does not naturally give harmonic resonances so as to approximate a harmonic series: this is the case with xylophone or vibraphone bars, bells or tabla (see [1] for various examples). The case of the piano falls rather into the first category, but the historical development of this instrument has gradually moved it away from the category of instruments with harmonic resonators. In fact the linear mass of the strings was multiplied by 4 in the second half of the 19th century [2] and the inharmonicity is no longer negligible. This is indeed an important attribute of modern pianos [3] which has a direct effect on the tuning, the octaves being widened so as to limit the beats between the different partials [4]. Overall the inharmonicity increases with the pitch of the note but in the first 2 octaves this inharmonicity increases when the pitch of the note decreases $[5,6]$. This phenomenon is particularly marked on upright piano with strings around $1 \mathrm{~m}$ length. The tonal quality of the notes of the first octave is particularly poor and it is difficult to assign a pitch to the first notes [7]. This phenomenon is undoubtedly together with the mechanics, one of the reasons why professional pianists are turning away from upright pianos.

*Corresponding author: jean-pierre.dalmont@univ-lemans.fr
The strong inharmonicity of the piano bass strings seems to be considered by instruments makers as inevitable. However, as early as the 1949, Miller [8] proposed tracks to correct the inharmonicity of the piano strings. It do not seem that this theoretical work lead to practical implementations. In 1998, Sanderson [9] patented an overwound near the end of the string to compensate for the unwound parts of the string. Curiously, his intention was not to reduce as much as possible the inharmonicity but to achieve an "ideal inharmonicity". These strings are marketed as Sanderson Accu-String. In a recent paper, Kemp [10] applied about the same idea to the bass guitar. Modeling the string using finite difference time domain modelling as well as an analytical perturbation method he succeeded in reducing significantly the anharmonicity of the first partials. Experiments have been made which agree reasonably with modelling.

In a previous paper [11], present author and Maugeais, with an optimization algorithm, looked for geometry which would lead to perfectly harmonic modes. Without constraining the algorithm, it converges to a winding which linear density varies sinusoidally along the string with a large increase in amplitude near the ends (Fig. 1). The results are as follows:

(i) The number of oscillations along the length of the string is strictly equal to the number of partial that the algorithm is trying to optimize.

(ii) The amplitude of the oscillations is all the more important the higher the number of partials optimized.

(iii) There is a practical limit to the number of partial that can be corrected, this limit being a function of the initial inharmonicity. 


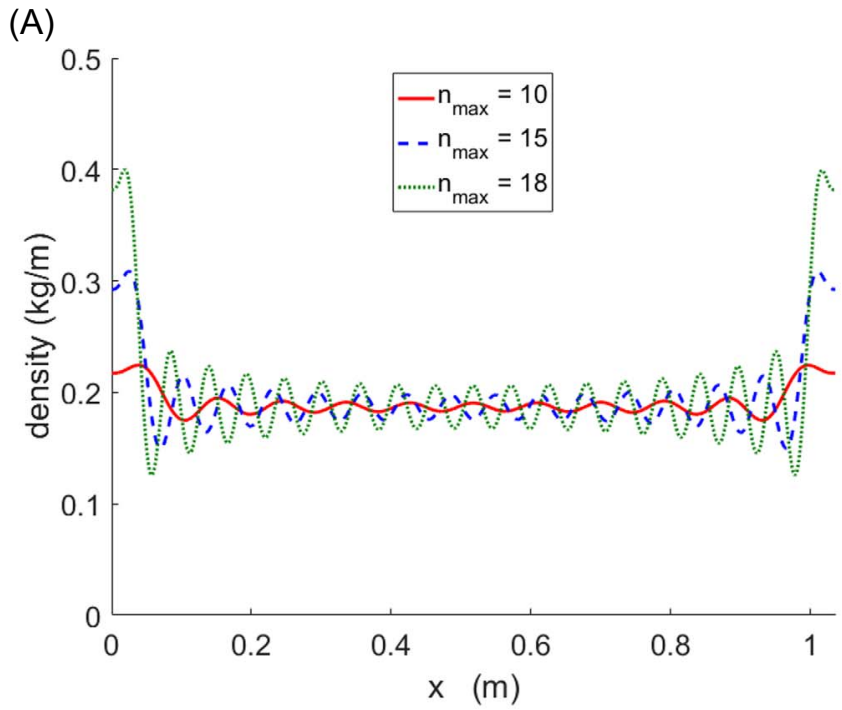

(B)

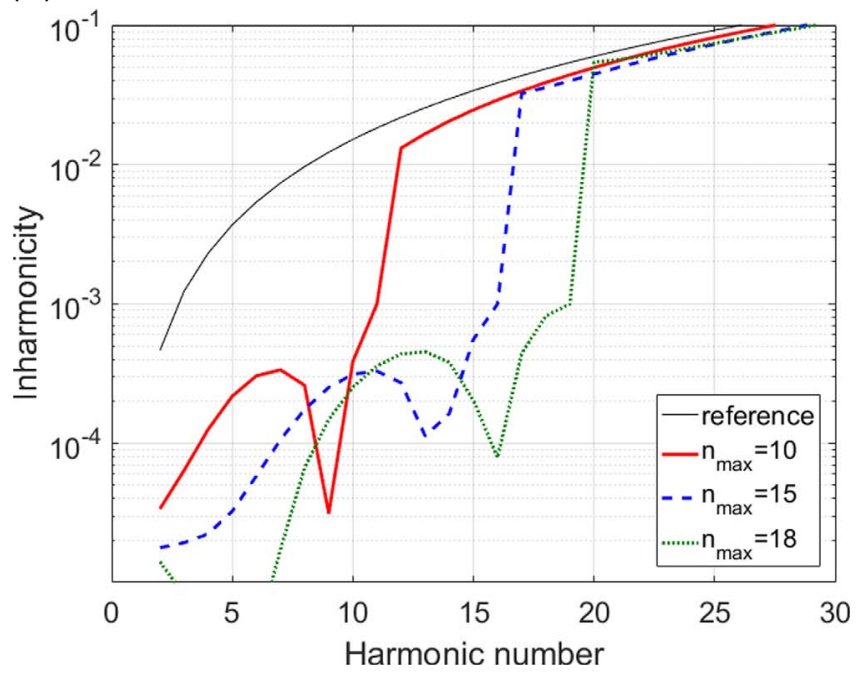

Figure 1. Result of an unconstrained optimization for a number of optimized harmonics equal to 10, 15 and 18 for a $1 \mathrm{~m}$ string such that the inharmonicity factor (Eq. (1)) is $B=3.13 \times 10^{-4}$ (from [11]). (A) Linear density versus position on the string. (B) Inharmonicity $I=1-\frac{f_{n}}{n f_{1}}$ as a function of partial number.

One of these results is rather frustrating since starting from a string with a strong inharmonicity, it will not be possible to reduce the inharmonicity on a large number of partials. The improvement will therefore necessarily be limited, especially if the bending stiffness is high.

The objective of this paper is twofold: on the one hand, to try to explain the results of the optimization on the basis of analytical formulas; on the other hand to develop a method making it possible to significantly reduce inharmonicity and to implement it on an upright piano.

In the first part we show how the solution resulting from the optimization combines two complementary correction strategies, added mass and sinusoidally varying density. In a second part we describe the implementation of the correction with an added mass and analyze the results of this correction on a real piano.

\section{Theoretical considerations}

The bending stiffness in a string model induces a dispersion which increases with frequency (see [12] for a detailed analysis). This results in an inharmonicity of the natural frequencies given by the well-known formula,

$$
\frac{f_{n}}{n f_{o}}=\sqrt{1+B n^{2}},
$$

with $f_{0}=\frac{c_{0}}{2 L}$ where $L$ is the length of the string, $c_{0}=\sqrt{\frac{T}{\mu}}$ the speed of the waves in a string without stiffness with $T$ the tension and $\mu$ the linear density and $B=\frac{\pi^{2} E I_{0}}{T L^{2}}$ where $E$ is the young modulus and $I_{0}$ the second moment of area of the cross section of the core considering that the winding does not contribute to the stiffness [13].

The coefficient $B$ is typically in the order of $10^{-4}$ for the strings of the middle of the instrument but in the order of $10^{-2}$ for the highest notes [6]. The inharmonicity of the treble strings, in addition to their metallic tone, involves widening the octaves very significantly in the treble [14]. For the lowest strings $B$ is near $10^{-4}$ for a large grand piano but can reach $10^{-3}$ for an upright piano [6]. The consequence is an unpleasant tone and an ill-defined pitch [7].

Unconstrained optimization (Fig. 1) suggests the combination of two strategies: an added mass near the edge and a periodic fluctuation in density along the string.

\subsection{Point mass}

The addition of a mass $m$ at a distance $a$ from the end of a string without stiffness breaks to the natural harmonicity of the eigenmodes. This can be calculated using the concept of equivalent length. For a uniform string of length $L$ without stiffness fixed at one end, the mechanical impedance $Z_{u}$ at the other end is given by,

$$
Z_{u}=-j Z_{c} \cot k L,
$$

where $k=\sqrt{\frac{T}{\mu}}$ is the wave number and $Z_{c}=\sqrt{\mu T}$ the characteristic impedance.

For a non uniform string or a string with some defects whose impedance is $Z_{n u}$, the equivalent length is then defined writing,

$$
Z_{n u}=-j Z_{c} \cot k L_{\mathrm{eq}},
$$

where $k$ and $Z_{c}$ are that of the uniform string.

Considering a portion of string of length $a$ with a mass $m$ at this point (Fig. 2), the impedance $Z_{a}$ is given by,

$$
Z_{a}=j m \omega-j Z_{c} \cot k a,
$$

and, considering $k=\frac{\omega}{c}$, with $c=\sqrt{\frac{T}{\mu}}$,

$$
a_{\text {eq }}=\frac{1}{k} \operatorname{atan}\left(\left(\cot k a-\frac{m}{\mu a} k a\right)^{-1}\right) \text {. }
$$




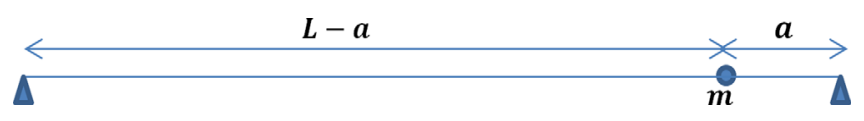

Figure 2. Scheme of a string of length $\boldsymbol{L}$ with a mass $\boldsymbol{m}$ at a distance $\boldsymbol{a}$ from the end.

For the whole string the equivalent length is then given by,

$$
L_{\mathrm{eq}}=L-a+a_{\mathrm{eq}} .
$$

The equivalent length is frequency dependent and can be related to the inharmonicity induced by the mass writing $-I(\omega)=1-\frac{L}{L_{\mathrm{eq}}}$. This is plotted Figure 3 for $L=1 \mathrm{~m}$, $m=5 \mathrm{~g}, a=2.4 \mathrm{~cm}$ and $\mu=180 \mathrm{~g} / \mathrm{m}$.

The inharmonicity is negative which means that the mass decreases the frequencies of the harmonics. It is null when $a$ is a multiple of the half wavelength. When $k a \ll 1$, a parabolic approximation can be made considering $\tan k a \cong k a \cong$ atan $k a$. Parameter $\frac{m}{\mu a}$ being of the order of unity, the equivalent length at second order is then,

$$
a_{\mathrm{eq}} \cong a\left(1+\frac{m}{\mu a}(k a)^{2}\right)
$$

and as well,

$$
1-\frac{L}{L_{\mathrm{eq}}} \cong \frac{m}{\mu L}(k a)^{2} .
$$

It can be seen that the effect of mass is at first approximation opposite to that of the stiffness of the string. So, when the condition,

$$
B \approx 2 \frac{m}{\mu L} \frac{\pi^{2} a^{2}}{L^{2}}
$$

is fulfilled and for harmonics such that $k a \ll 1$, an added mass can therefore compensate for the inharmonicity due to the stiffness of the string. Let consider for example a $1 \mathrm{~m}$ string such that $B=3.13 \times 10^{-4}$, with a linear mass $\mu=0.18 \mathrm{~kg} / \mathrm{m}$. If we choose to place a mass $m=5 \mathrm{~g}$, this one has to be placed $2.4 \mathrm{~cm}$ from the end. Effect of mass decreases rapidly beyond the 13th harmonic for which half wave length is $7.7 \mathrm{~cm}$ and only partially compensates for the inharmonicity, becoming negligible (see Fig. 3). For a mass placed beyond $3 \mathrm{~cm}$, the number of harmonics effectively treated is less than 10 . It is therefore necessary to place the mass as close as possible to the attachment, knowing that the added mass has to be greater when it is close to the end. Placing the mass between 2 and $3 \%$ of the length of the string therefore seems to be the most reasonable solution for a string of the order of $1 \mathrm{~m}$.

\subsection{Periodic linear density fluctuation}

We consider a string which linear density $\mu$ varies sinusoidally along the string such that $\mu=\mu_{0}+\Delta \mu_{s} \sin \frac{2 \pi x}{b}$ where $b$ is the fluctuation period, $\Delta \mu_{s}$ the amplitude of the fluctuation and $\mu_{0}$ the mean value. For low frequencies, periodic mass fluctuation is a second order effect in which the amplitude of the fluctuations occurs to the power 2

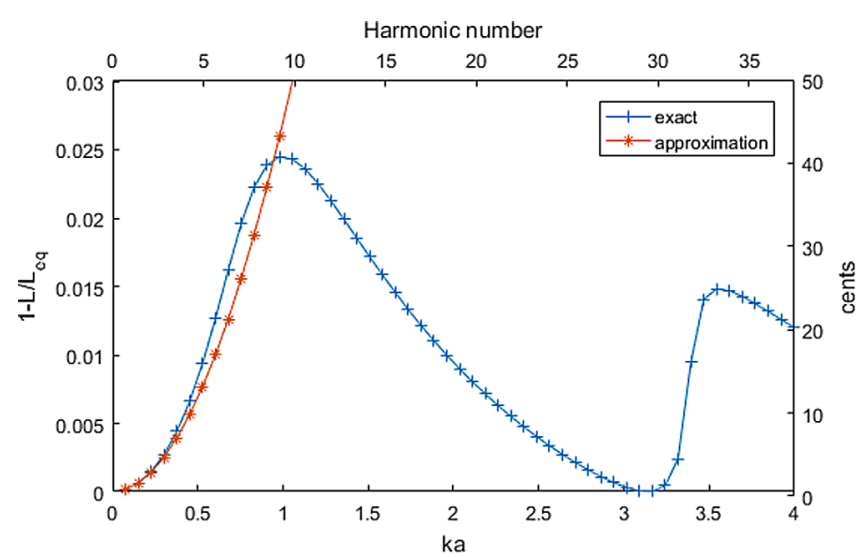

Figure 3. $1-\frac{L}{L_{e q}}=-I$ as a function of $k a$ for $L=1 \mathrm{~m}$, $\mu=180 \mathrm{~g} / \mathrm{m}, a \stackrel{L_{e q}}{=} 2.4 \mathrm{~cm}$ and $m=5 \mathrm{~g}$ (Eqs. (6) and (8) for the approximation). Harmonics for which $1-\frac{L}{L_{e q}}=1-\frac{f_{n}}{n f_{0}}=-I_{n}$ are marked with crosses and stars and harmonic number is indicated with an upper scale. Right scale in cents is for $1200 \frac{\log \frac{L_{e q}}{L}}{\log 2}$.

(see Appendix). For the low values of $k b(b \ll \lambda)$, a first order development can be done and the rate of fluctuation amplitude $\frac{\Delta \mu_{s}}{\mu_{0}}$ which would compensate for the natural inharmonicity of the rigid string can be deduced: $\frac{\Delta \mu_{s}}{\mu_{0}} \approx 4 L / b \sqrt{\frac{3 B}{2 \pi}}$. This rate of fluctuation is proportional to the square root of $B$ but also to the number of density oscillations on the string $N=L / b$. The number of oscillations will necessarily be limited because the rate of fluctuation being proportional to the number of oscillations, this one can theoretically become greater than unity, which is in practice not possible. For example for $B=3.13 \times 10^{-4}$ and $N=30$, we obtain $\frac{\Delta \mu_{s}}{\mu_{0}}=1.47$ which is not feasible. But the main difficulty comes from the fact that beyond $k b>\frac{\pi}{2}$, the effect of the fluctuations increases considerably until the frequency reaches a stop band for which waves no longer propagate. This solution therefore generates a high inharmonicity in the vicinity of $k b=\pi$ (Fig. 4).

Density fluctuations can therefore not be retained as a solution for reducing inharmonicity but might be used as a complement to a point mass. Indeed, the optimization without constraint converges towards a periodic fluctuation of density with a strong increase near the end which can be interpreted as the combination of the two solutions examined previously: periodic fluctuation of density and point mass near the end.

In Figure 5 is shown the combined effect of a point mass and a density fluctuation. The two solutions complement each other in terms of frequency, but, as Miller [8] notes with reference to Rayleigh, the periodic oscillation acts essentially on a frequency $(k b \approx \pi)$. The density fluctuation thus appears rather as an artifact of the simulation algorithm because it makes it possible to reach very low values of inharmonicity which do not have any practical interest. Moreover, it tends to degrade the harmonicity beyond the last optimized harmonic. Given its difficulty of implementation and its low added value, the track of density fluctuation was therefore abandoned for the experiments. 


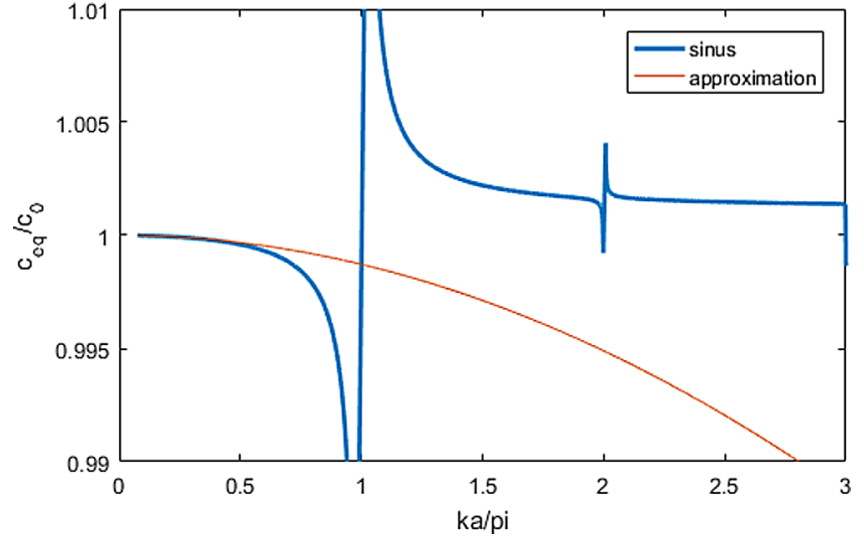

Figure 4. $\frac{c_{\mathrm{eq}}}{c_{0}}=\frac{k_{0}}{k_{\mathrm{eq}}}$ as a function of $\frac{k b}{\pi}$ for sinusoidal linear density fluctuations with $\frac{\Delta \mu_{s}}{\mu_{0}}=0.14$ and $b=7.5 \mathrm{~cm}$. Thin red line: parabolic approximation.

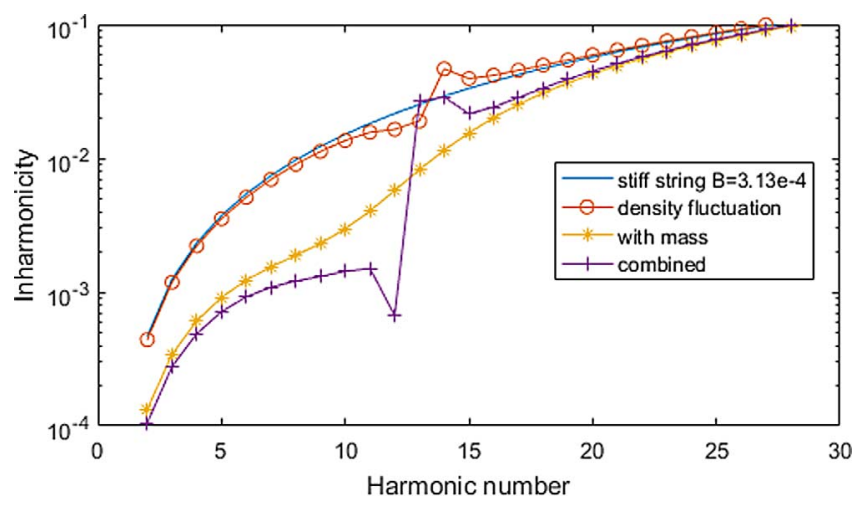

Figure 5. Inharmonicity as a function of harmonic number for a string of length $L=1 \mathrm{~m}$ and with a linear density $\mu=180 \mathrm{~g} / \mathrm{m}$. Blue curve: string alone with $B=3.13 \times 10^{-4}$. Red curve: string with sinusoidal density fluctuations such that $\frac{\Delta \mu_{s}}{\mu_{0}}=0.14$ and $b=7.5 \mathrm{~cm}$ (see Appendix). Yellow curve: string with a mass $m=3.5 \mathrm{~g}$ at $a=2.4 \mathrm{~cm}$ from the end. Purple curve: string with a mass $(m=3.5 \mathrm{~g}$ and $a=2.4 \mathrm{~cm})$ and sinusoidal density fluctuations $\left(\frac{\Delta \mu_{s}}{\mu_{0}}=0.14\right.$ and $\left.b=7.5 \mathrm{~cm}\right)$.

\section{Experiments}

As discussed in the previous section, adding masses seems to be a promising solution. In practice, difficulty consists in fixing a mass which do not add damping nor oscillates itself. From this point of view the use of magnets has been found to be unsatisfactory. Finally we opted for split spherical fishing sinkers glued with cyanoacrylate glue. Sanderson [9] used strings where a winding was applied towards the end of the string and then reversed over the rest of the string to leave an increased mass near the piano string end. Kemp [10] used a separate layer of winding of smaller cross-section placed under the main winding(s) of bass guitar strings. The risk with over winding is that it may increase locally the stiffness which may counterbalance the desired effect.

We worked on a Yamaha C109 piano (no. J2299073) and limited our work to single strings. These range from

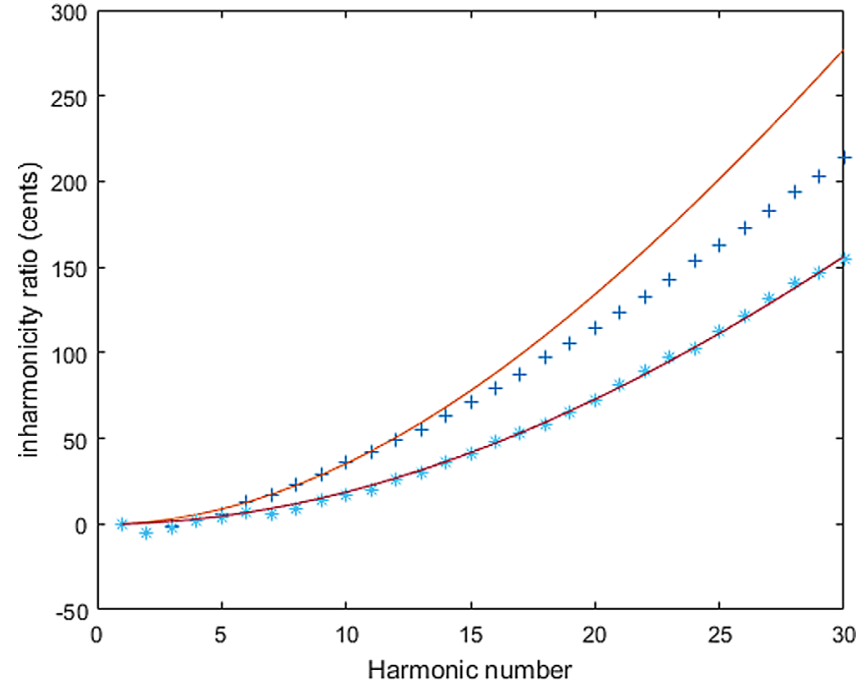

Figure 6. Inharmonicity ratio $\frac{f_{n}}{n f_{1}}$ (cents) versus harmonic number. +: C0; *: A0. Continuous curves: $\frac{\sqrt{1+B n^{2}}}{\sqrt{1+B}}$ with $B=4.2 \times 10^{-4}$ and $B=2.2 \times 10^{-4}$ respectively.

A-1 to A0. They are double wound except for $\mathrm{G} \# 0$ and A0. The strings have an unwound part on approximately $1.5 \mathrm{~cm}$ then a part with a simple winding on several $\mathrm{cm}$ (see Fig. 8). The masses are therefore glued to the single wound part between 1.5 and $3 \mathrm{~cm}$ from the end. It is worth mentioning that the unwound part increases inharmonicity as shown in previous research by Chumnantas [15]. The correction due to this is likely to be larger for the double wound string. The added mass shall also compensate for this.

We first recorded the spectra of the strings without added mass. It appears that the inharmonicity curves of double-wound strings do not follow the theoretical law (Eq. (1)) beyond harmonic 12, while the single-wound strings follow it perfectly (Fig. 5).

The value of $B$ is then determined as the average on the partials $6-12$ because beyond 12 the evolution is no longer parabolic and below 6 the evaluation of $B$ is affected by a great uncertainty (Fig. 6). Part of this uncertainty is due to the fact that we used a simple Fourier transform for which the frequency resolution is the invert of the time duration of the recording (about $16 \mathrm{~s}$ which corresponds to 2 cents for A0). The value of $B$ decreases regularly from $4.5 \times 10^{-4}$ for $\mathrm{Bb}-1$ to $3.2 \times 10^{-4}$ for G0. The A-1 is apart with $B=5.75 \times 10^{-4}$. Also, G\# 1 and A1 have a lower coefficient of inharmonicity $B$ on partial $6-12$ but they follow the parabolic law, the inharmonicity of the higher harmonics will then be closer to that of the other strings (Fig. 7).

The location of the masses is determined from Equation (9), the masses of 10,5 and $2.5 \mathrm{~g}$ being chosen so as not to exceed $3 \mathrm{~cm}$. We thus obtain $10 \mathrm{~g}$ masses for $\mathrm{A}-1$ and $\mathrm{B}-1$ (for practical reasons $\mathrm{Bb}-1$ has not been treated), $5 \mathrm{~g}$ for $\mathrm{C} 0-\mathrm{E} 0$ and $2.5 \mathrm{~g}$ for $\mathrm{F} 0-\mathrm{A} 0$. The theoretical position of each mass is given in Figure 8. 


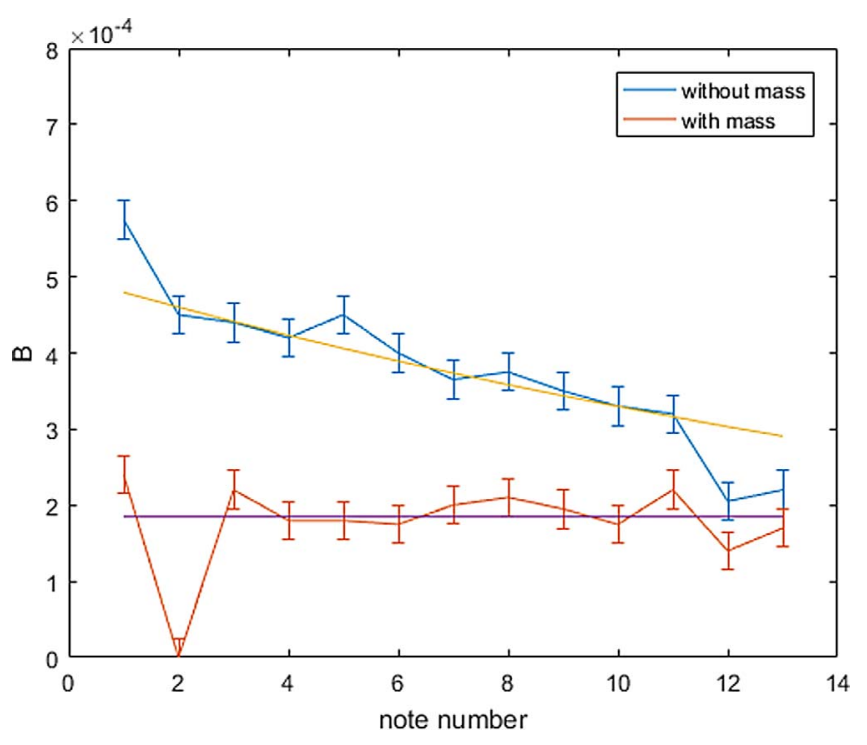

Figure 7. Inharmonicity coefficient $B$ versus note number with (higher curve) and without (lower curve) added mass.

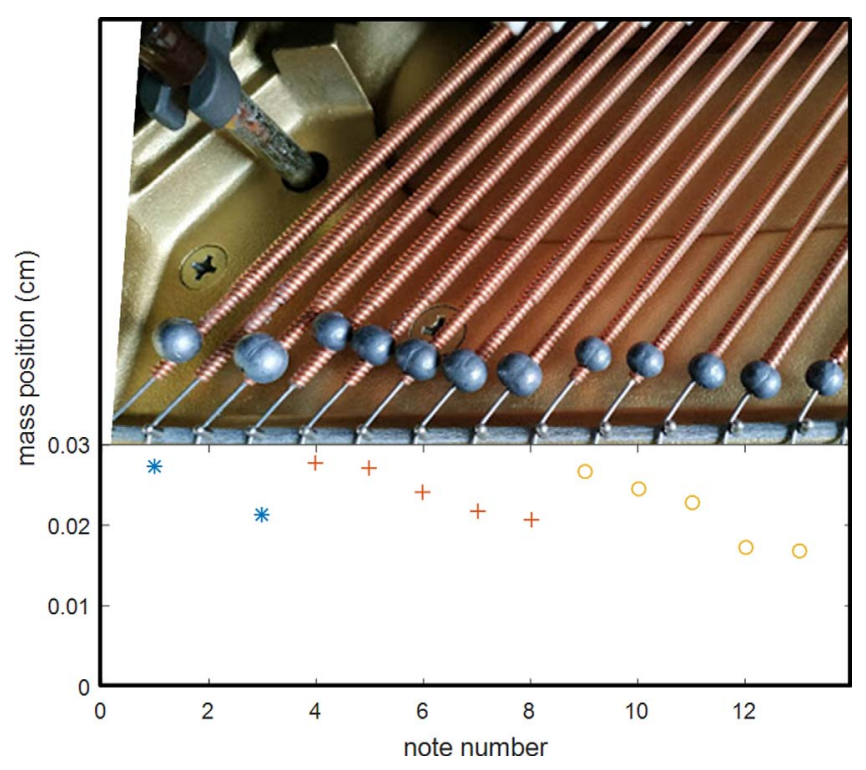

Figure 8. Theoretical location of added masses for each note (* $10 \mathrm{~g} ;+5 \mathrm{~g}$; o $2.5 \mathrm{~g}$ ). Picture: effective location of the masses (the image has been turned upside down to allow comparison with the curve).

The inharmonicity of each note is determined according to the same procedure as above and the values of $B$ obtained show a significant reduction in the inharmonicity. The mean value for $B$ is now $1.85 \mathrm{e}-4$ (Fig. 7 ). The inharmonicity curve tends to join that of the string without mass for high order harmonics greater than 30 (Fig. 9). It should be noted that the spectrum with mass is less extended than that of the strings without mass which can also have a great influence on the perceived timbre (see Galembo et al. [16]).

The sounds can be heard in the files "Do0 to La0 without mass.wav" and "Do0 to La0 with mass.wav". In another

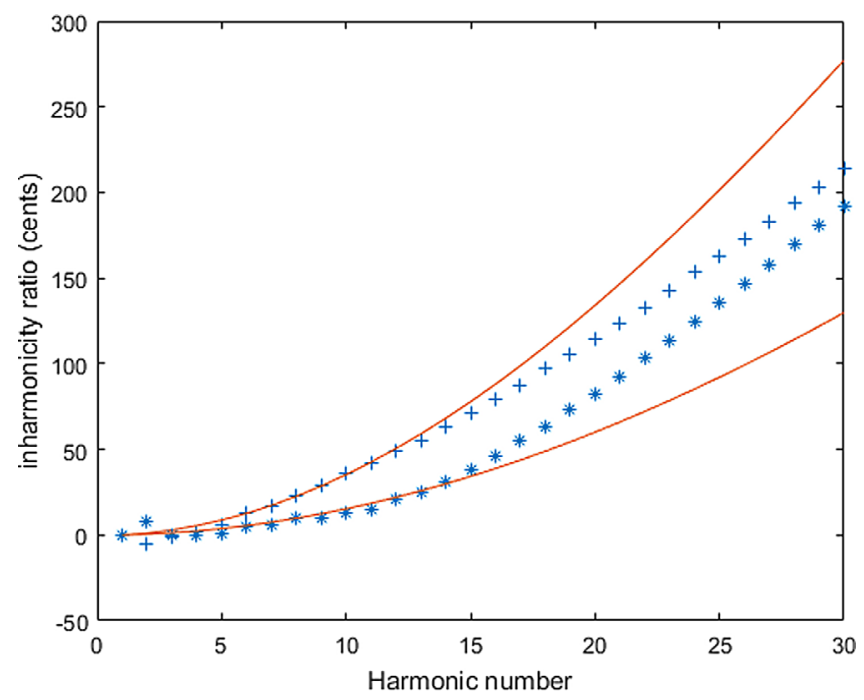

Figure 9. Inharmonicity ratio $\frac{f_{n}}{n f_{1}}$ (cents) versus harmonic number. +: $\mathrm{C} 0$ without mass; *: $\mathrm{C} 0$ with mass. Continuous curves: $\frac{\sqrt{1+B n^{2}}}{\sqrt{1+B}}$ with $B=4.2 \times 10^{-4}$ and $B=1.8 \times 10^{-4}$ respectively.

file, "Do0 to Sol0 without and with masse alternated. wav" the same sounds are given alternatively with mass (first) and without mass so as to facilitate comparison (Supplementary Material).

\section{Conclusion}

The study conducted here allows us to understand the results from the digital optimization proposed by Maugeais together with the present author in a previous paper [11]. This indeed seems schematically to combine a sinusoidal fluctuation in density and the addition of a point mass at the end of the string. The analytical study of these two solutions makes it possible to show on the one hand that the density fluctuations along the string potentially do little to improve the situation because it acts in a significant way only around one specific harmonic. On the other hand, the addition of a point mass makes it possible to effectively correct the inharmonicity of the first harmonics, the position and the value of the mass being linked to the value of the coefficient of inharmonicity $B$. The implementation of this solution is simple: after a measurement of the inharmonicity of each string it is easy to theoretically determine the position of each mass on the string, the value of the mass being chosen so as not to place the mass at more than $3 \mathrm{~cm}$ from the end. This ensures a significant reduction of the inharmonicity of the first 10 harmonics.

Experiments show that with masses of 10,5 and $2.5 \mathrm{~g}$ all the single strings can be corrected, bringing the inharmonicity to values typically half as low as for uncorrected strings. From a musical point of view we perceive a better definition of the pitch and a less metallic sound. Indeed as shown by Giordano [4] the uncertainty on the perception of the pitch is particularly high in the low frequencies: \pm 150 cents for 
the Do0 on an upright piano. A psychoacoustic study should be carried out extending that of Giordano in order to determine how the perceived pitch is influenced by the inharmonicity and how the differential frequency threshold is itself influenced. In addition, we should work with piano makers and musicians to assess to which extent the reduction in the inharmonicity of the first partials is perceived as an improvement and can be implemented in the long term on given pianos. It must indeed be recognized that even if the sound and the pitch definition of the first notes are improved, the inharmonicity of high order partials is still quite large and the sound quality remains lower than that of a grand piano. It would be interesting to work on halfgrand pianos for which the first notes are less dramatically inharmonic but nevertheless deserve to be treated.

\section{Acknowledgments}

We thank Sylvain Maugeais, Frédéric Ablitzer, Philippe Béquin for fruitful discussions and Patrick Sinigaglia and the Itemm for the provision of the piano and assistance with the experiments. We also thank reviewers for their valuable comments and suggestions.

\section{Conflict of interest}

Author declared no conflict of interests.

\section{Supplementary material}

Supplementary material is available at https://actaacustica.edpsciences.org/10.1051/aacus/2021002/olm

Three sound files can be heard: "Do0 to La0 without mass.wav" and "Do0 to La0 with mass.wav". In another file, "Do0 to Sol0 without and with masse alternated.wav" the same sounds are given alternatively with mass (first) and without mass so as to facilitate comparison.

Supplementary File 1. Do0 to La0 with mass.wav

Supplementary File 2. Do0 to La0 without mass.wav

Supplementary File 3. Do0 to Sol0 without and with masse alternated.wav

\section{References}

1. N. Fletcher, T. Rossing: Physics of musical instruments. 2nd ed., Springer, 1998.

2. A. Chaigne: The making of pianos: a historical view. Musique et Techniques 8 (2017), Itemm.

3. H. Fletcher, E.D. Blackham, R. Stratton: Quality of piano tones. The Journal of the Acoustical Society of America 34 (1962) 749-761.

4. N. Giordano: Explaining the railsback stretch in terms of the inharmonicity of piano tones and sensory dissonance. The Journal of the Acoustical Society of America 138 (2015) $2359-2366$.

5. F. Rigaud, B. David, L. Daudet: A parametric model and estimation techniques for the inharmonicity and tuning of the piano. The Journal of the Acoustical Society of America 133 (2013) 3107-3118.

6. H.A. Conklin: Design and tone in the mechanoacoustic piano. part III. piano strings and scale design. The Journal of the Acoustical Society of America 100 (1996) 1286-1298.

7.B.E. Anderson, W.J. Strong: The effect of inharmonic partials on pitch of piano tones. The Journal of the Acoustical Society of America 117 (2005) 3268-3272.

8. F. Miller: A proposed loading of piano strings for improved tone. The Journal of the Acoustical Society of America 21 (1949) 318-322.

9. A.E. Sanderson: Method for making wound strings for musical instruments characterized by reduced inharmonicity. US Patent 5984226 (1999).

10. J.A. Kemp: On inharmonicity in bass guitar strings with application to tapered and lumped constructions. SN Applied Sciences 2 (2020) 636 .

11. J.P. Dalmont, S. Maugeais: Piano strings with reduced inharmonicity. Acta Acustica United With Acustica, Hirzel Verlag 105 (2019) 714-726.

12. E. Ducasse: On waveguide modeling of stiff piano strings. The Journal of the Acoustical Society of America 118 (2005) 1776-1781.

13. J. Chabassier: Modeling and numerical simulation of a piano (in French). PhD thesis, Ecole Polytechnique, 2012.

14. R.A. Rasch, V. Heetvelt: String inharmonicity and piano tuning. Music Perception: An Interdisciplinary Journal 3, 2 (1985) 171-189.

15. P. Chumnantas: Inharmonicity in the natural mode frequencies of overwound strings. PhD Dissertation, The University of Edinburgh, 1995.

16. A. Galembo, A. Askenfelt, L.L. Cuddy, F.A. Russo: Perceptual relevance of inharmonicity and spectral envelope in the piano bass range. Acta Acustica United With Acustica, Hirzel Verlag 90 (2004) 528-536.

17. L. Brillouin, M. Parodi: Wave Propagation in Periodic Structures. Mc Graw Hill, New York, NY, USA, 1946.

\section{Appendix}

\section{Propagation in a string of periodically variable density}

Consider a string without stiffness whose linear mass $\mu$ varies periodically in the form,

$$
\mu(x)=\mu_{0}+\Delta \mu \operatorname{sign}\left(\sin \frac{2 \pi x}{b}\right)
$$

where $\mu_{0}$ is the average linear mass, $\Delta \mu$ the amplitude of the density fluctuation and $b$ the spatial period of fluctuation (see Fig. A.1).

A spatial period therefore consists of two portions of strings of length equal to $b / 2$. For these two portions of strings, the characteristic impedances are given by,

$$
Z_{c \pm}=\sqrt{\mu_{ \pm} T}
$$

with $\mu_{ \pm}=\mu_{0} \pm \Delta \mu$ and the wave numbers by,

$$
k_{ \pm}=\frac{\omega}{c_{ \pm}},
$$

where $c_{ \pm}=\sqrt{\frac{T}{\mu_{ \pm}}}$.

The transfer matrix for a fluctuation period is therefore written, 


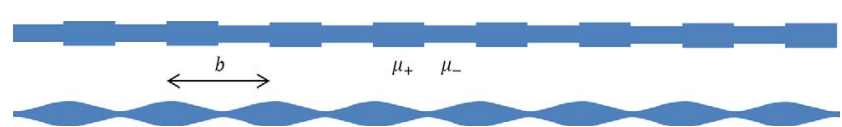

Figure A.1. Schematic view of a string with a square variation of the linear density and of a string with a sinusoidal variation of density

\section{See Equation (A.4) bottom of this page}

The network propagation constant $k_{\text {eq }}$ can be deduced from the previous transfer matrix setting [17],

$$
\frac{A+D}{2}=\cos \left(k_{\mathrm{eq}} b\right) \text {. }
$$

We have,

See Equation (A.6) bottom of this page

or else,

$$
\begin{aligned}
\frac{A+D}{2}= & \cos \left(\frac{k_{+} b+k_{-} b}{2}\right)-\frac{\left(Z_{c+}-Z_{c-}\right)^{2}}{2 Z_{c+} Z_{c-}} \\
& \times \sin \left(\frac{k_{+} b}{2}\right) \sin \left(\frac{k_{-} b}{2}\right),
\end{aligned}
$$

then,

$k_{\mathrm{eq}}=\frac{1}{b} \operatorname{acos}\left[\cos \left(\frac{k_{+} b+k_{-} b}{2}\right)-\frac{\left(Z_{c+}-Z_{c-}\right)^{2}}{2 Z_{c+} Z_{c-}} \sin \left(\frac{k_{+} b}{2}\right) \sin \left(\frac{k_{-} b}{2}\right)\right]$.

The function $k_{\mathrm{eq}}$ is given Figure A.2.

We also consider a string without stiffness whose linear mass $\mu$ varies periodically in the form,

$$
\mu=\mu_{0}+\Delta \mu_{s} \sin \frac{2 \pi x}{b},
$$

with $\Delta \mu_{s}$ the amplitude of the linear mass fluctuation.

When calculating numerically the propagation constant with the Transmission Matrix Method (TMM) on 1000 slices, a dispersion diagram almost identical to the previous one is obtained setting,

$$
\Delta \mu_{s}=\sqrt{\frac{\pi}{2}} \Delta \mu
$$

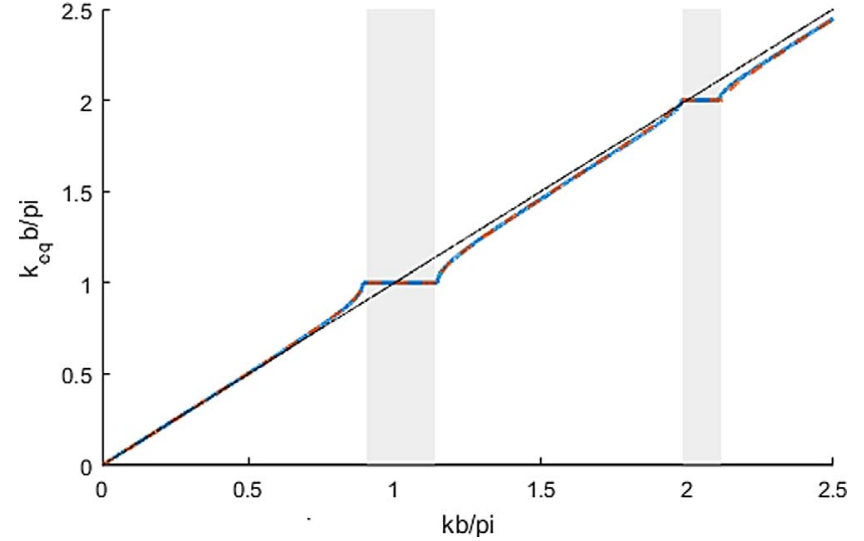

Figure A.2. Dispersion diagram of a string with periodically variable linear density. Blue curve: sinusoidal density fluctuation with $\Delta \mu_{s}=0.5$. Dotted orange curve: square density fluctuation with $\Delta \mu=0.5 \sqrt{\frac{2}{\pi}}$. Black line: uniform string $\left(k_{\mathrm{eq}}=k\right.$ with $\left.k=\sqrt{\frac{T}{\mu_{0}}}\right)$. Grey bars: stop bands.

where $\Delta \mu_{s}$ is the amplitude of the sinusoidal fluctuation and $\Delta \mu$ that of the equivalent square fluctuation. Deviation is less than $0.1 \%$ below the first cut-off frequency except in the vicinity of the cut-off frequency (see Fig. A.2).

Wave speed at low frequency is that of the uniform string. It decreases with frequency until the waves no longer propagate (stop band). This occurs when the argument of the function acos is equal to -1 which occurs when the half-wavelength is close to the fluctuation period and more precisely when,

$\left[\cos \left(\frac{k_{+} b+k_{-} b}{2}\right)-\frac{\left(Z_{c+}-Z_{c-}\right)^{2}}{2 Z_{c+} Z_{c-}} \sin \left(\frac{k_{+} b}{2}\right) \sin \left(\frac{k_{-} b}{2}\right)\right]=-1$.

The phenomenon occurs all the sooner than $\frac{\Delta \mu}{\mu_{0}} \ll 1$ is large. Assuming that $\frac{\Delta \mu}{\mu_{0}} \ll 1$ the limits of the first stop band is obtained approximately when,

$$
k_{0} b \approx \pi \pm \frac{\Delta \mu}{\mu_{0}},
$$

with $k_{0}=\frac{\omega}{c_{0}}$ where $c_{0}=\sqrt{\frac{T}{\mu_{0}}}$.

At low frequencies, the wave speed can be approximated as a function of frequency by the inverse of a second degree polynomial:

$$
\begin{gathered}
\left(\begin{array}{ll}
A & B \\
C & D
\end{array}\right)=\left(\begin{array}{cc}
\cos \left(\frac{k_{+} b}{2}\right) & j Z_{c+} \sin \left(\frac{k_{+} b}{2}\right) \\
j Z_{c+}^{-1} \sin \left(\frac{k_{+} b}{2}\right) & \cos \left(\frac{k_{+} b}{2}\right)
\end{array}\right)\left(\begin{array}{cc}
\cos \left(\frac{k_{-} b}{2}\right) & j Z_{c-} \sin \left(\frac{k_{-} b}{2}\right) \\
j Z_{c-}^{-1} \sin \left(\frac{k_{-} b}{2}\right) & \cos \left(\frac{k_{-} b}{2}\right)
\end{array}\right) . \\
\frac{A+D}{2}=\cos \left(\frac{k_{+} b}{2}\right) \cos \left(\frac{k_{-} b}{2}\right)-\frac{1}{2}\left(\frac{Z_{c+}}{Z_{c-}}+\frac{Z_{c-}}{Z_{c+}}\right) \sin \left(\frac{k_{+} b}{2}\right) \sin \left(\frac{k_{-} b}{2}\right),
\end{gathered}
$$




$$
c_{\mathrm{eq}} \approx c_{0} /\left(1+\left(\frac{\Delta \mu}{2 \mu_{0}}\right)^{2} \frac{\left(k_{0} b\right)^{2}}{24}\right)
$$

The effect is therefore similar to that of stiffness but of opposite sign. The two effects may compensate at low frequency if,

$$
\frac{B n^{2}}{2}=\left(\frac{\Delta \mu}{2 \mu_{0}}\right)^{2} \frac{\left(k_{0} a\right)^{2}}{24},
$$

i.e.,

$$
B \approx \frac{1}{3}\left(\frac{\pi \Delta \mu b}{4 \mu_{0} L}\right)^{2}
$$

or, for a sinusoidal modulation of amplitude $\Delta \mu_{s}$, according to Equation (A.10),

$$
B \approx \frac{2 \pi}{3}\left(\frac{\Delta \mu_{s} b}{4 \mu_{0} L}\right)^{2} .
$$

Cite this article as: Dalmont J.-P. 2021. Piano bass strings with reduced inharmonicity: theory and experiments. Acta Acustica, 5, 9 . 